\title{
BMJ Open Individual cognitive stimulation therapy for people with intellectual disability and dementia: protocol of a feasibility randomised controlled trial
}

\author{
Afia Ali, ${ }^{1}$ Emma Brown, ${ }^{1}$ Aimee Spector, ${ }^{2}$ Elisa Aguirre, ${ }^{3}$ Angela Hassiotis ${ }^{1}$
}

To cite: Ali A, Brown E, Spector A, et al. Individual cognitive stimulation therapy for people with intellectual disability and dementia: protocol of a feasibility randomised controlled trial. BMJ Open 2018;8:e022136. doi:10.1136/ bmjopen-2018-022136

- Prepublication history for this paper is available online. To view these files, please visit the journal online (http://dx.doi org/10.1136/bmjopen-2018022136).

Received 6 February 2018

Revised 4 June 2018

Accepted 24 October 2018

Check for updates

(c) Author(s) (or their employer(s)) 2018. Re-use permitted under CC BY-NC. No commercial re-use. See rights and permissions. Published by BMJ.

${ }^{1}$ Division of Psychiatry, University College London, London, UK

${ }^{2}$ Clinical, Education and Health Psychology, Division of Psychology and Language Sciences, University College London, London, UK

${ }^{3}$ Talking Therapies, Barking and Dagenham IAPT, NHS Foundation trust, llford, UK

Correspondence to

Dr Afia Ali; afia.ali@ucl.ac.uk

\section{ABSTRACT}

Introduction Cognitive stimulation therapy (CST) is a psychosocial intervention for dementia. Group CST is effective in reducing cognitive decline and improving quality of life in patients with dementia. There is some evidence that individual CST (iCST) may be beneficial in reducing cognitive decline. People with intellectual disability (ID) have an increased risk of dementia. However, there are no published studies of CST in people with ID and dementia. This protocol describes the feasibility and acceptability of a randomised controlled trial of iCST delivered by carers to people with ID and dementia, compared with treatment as usual (TAU). The results of this study will inform the design of a future definitive randomised controlled trial.

Methods and analysis The iCST intervention has been adapted for this trial. Forty dyads (individuals with ID and their carer) will be randomised to either iCST or TAU. The manualised intervention comprises 40 iCST sessions delivered by a carer for $30 \mathrm{~min}$, twice a week, over 20 weeks. The primary outcome will be process measures assessing the feasibility and acceptability of the intervention and trial procedures. The secondary outcome will be changes in the scores of outcome measures (cognition, functional ability and quality of life in individuals with ID, and caregiver burden, competence in managing dementia, and anxiety and depression in carers). Data will be collected at baseline, 11 weeks and at 21 weeks. A process evaluation will examine adherence to iCST and will include qualitative interviews with participants to identify aspects of the intervention that were or were not successful.

Ethics and dissemination The study has received ethical approval. The results of the study will be presented at conferences and submitted to a peer reviewed journal. Trial registration number ISRCTN18312288; Pre-results.

\section{INTRODUCTION}

The incidence of dementia in older people with intellectual disabilities (IDs) is almost five times higher compared with the general population. ${ }^{1}$ In people with Down syndrome, the risk of dementia is greatly increased. One study found that over $97 \%$ of participants with Down Syndrome developed Alzheimer's
Strengths and limitations of this study

This is the first feasibility randomised controlled trial on cognitive stimulation therapy which has been adapted for use in people with dementia and intellectual disability (ID).

- The study is being led by researchers with expertise in carrying out trials in people with ID and trials of cognitive stimulation therapy.

- The findings of the study will need to be interpreted with caution due to this being a feasibility study.

dementia over a 20 -year period. ${ }^{2}$ Dementia is a significant cause of morbidity and mortality in people with ID. ${ }^{3}$

There are several non-pharmacological interventions that aim to enhance cognition or reduce the impact of cognitive deficits in individuals suffering from dementia, including cognitive training, cognitive rehabilitation, reminiscence therapy and cognitive stimulation therapy (CST). Cognitive training involves guided practice on standardised tests that reflect specific cognitive functions such as attention, memory and problem solving. Currently, there is no evidence for the effectiveness of cognitive training on patients on dementia. ${ }^{4}$ Cognitive rehabilitation is an approach to managing the impact of dementia related symptoms, such as memory loss, on activities of daily living. It involves setting specific goals and using strategies to learn new information and compensatory techniques. There is evidence from a small randomised controlled trial that cognitive rehabilitation therapy may improve goal performance and subjective memory ratings in participants with dementia. ${ }^{5}$ Reminiscence therapy involves the discussion of past activities and experiences using prompts and props such as photographs or objects. There is evidence that reminiscence therapy 
may improve communication but its effects on cognition are small. ${ }^{6}$

CST uses a range of methods to stimulate learning and memory, including errorless learning, reality orientation and multisensory stimulation. ${ }^{78}$ Reality orientation involves presenting information about time, place and person to an individual in order to orient the individual to his/her environment. It has been criticised for being too rigid and confrontational. CST employs the positive aspects of reality orientation, using a sensitive, respectful and person centred approach. There is consistent evidence that CST improves cognitive functioning, quality of life, well-being, communication and social interaction in people with dementia in the general population. ${ }^{9}$ Its effects are most marked on language skills such as naming, word finding and comprehension. ${ }^{10}$ The benefits of CST may arise from activation of neuronal networks associated with cognition such as memory and language ${ }^{11}$ Most of the evidence is based on group CST, typically two sessions a week lasting $45 \mathrm{~min}$, over a 7 -week period. ${ }^{12}$ The intervention involves activities that include word association, categorisation, reminiscence, creative activities, number and word games and discussion of current affairs.

In the UK, the National Institute for Health and Clinical Excellence guidelines ${ }^{13}$ recommend that people with mild/moderate dementia should be given the opportunity to participate in a structured group CST programme. CST is cost-effective and has comparable efficacy to antidementia drugs. ${ }^{14}$

There have been no randomised controlled trials of CST or any of the aforementioned cognitive interventions in people with dementia and ID. Given that CST has the most evidence for improving cognition in the general population, it may have similar effects in other populations such as people with ID. However, people with ID may respond differently to the intervention due to the presence of premorbid cognitive difficulties and differences in their cognitive profile, and therefore it is imperative that the impact of CST is examined within a randomised controlled trial.

To our knowledge, there has been only one pilot randomised controlled study of 25 participants with Down syndrome without dementia investigating the impact of group CST in improving cognition, adaptive functioning and quality of life, compared with treatment as usual (TAU)..$^{15}$ The study found that the intervention significantly improved cognitive functioning in the group receiving CST pretreatment and post-treatment, and there was an improvement on quality of life scores at 3 months follow-up. However, when the treatment and control groups were compared, there were no differences in any of the outcomes post intervention and at 3 months. This finding is perhaps not surprising given that the participants did not have dementia and the sample size was also relatively small. However, the study did demonstrate that CST could be adapted for use in people with ID.
In order for group CST to be successful, the groups should comprise individuals with a similar degree of cognitive impairment in order to ensure that participants are effectively engaged. Differences in baseline cognition in individuals with ID, coupled with possible sensory impairment, poses a challenge for recruitment and effective group work. Individual CST (iCST) may therefore be a more practical and acceptable option for people with ID and dementia. iCST involves participating in one-to-one activities with a carer. The iCST programme is based on similar principles to group CST and involves mental stimulation, reminiscence and Reality Orientation. There are 10 principles: mental stimulation; developing new ideas, thoughts and associations; focusing on opinions rather than facts; using reminiscence; using triggers to support memory; using a 'person-centred' approach; offering a choice of activities; enjoyment and fun; maximising potential; and strengthening the relationship by spending quality time together. ${ }^{16}$ iCST therefore promotes positive interactions between the carer and individual which could benefit their relationship and potentially enhance cognition.

There is some evidence for the effectiveness of iCST delivered by carers, for people with dementia in the general population. Typically, 75 sessions are administered by carers over a 25-week period (three sessions, each $30 \mathrm{~min}$ ). A randomised controlled trial of individual reality orientation therapy in people with dementia receiving anticholinesterase inhibitors versus anticholinesterase inhibitors alone, found significant improvements in cognition but not for behavioural or functional outcomes. ${ }^{17}$ A recent multicentre randomised controlled trial of manualised iCST delivered by family carers, compared with TAU, in 356 carers and individuals with dementia ${ }^{18}$ found that iCST did not improve cognition or quality of life for people with dementia and it did not improve carers' physical or mental health. However, there was some improvement in the caregiving relationship and in carers' health related quality of life. Possible reasons for the lack of differences in the treatment and control groups in relation to cognition and quality of life could be attributed to the poor therapy adherence rate. Only $51 \%$ of the dyads completed more than 30 sessions out of 75 and $22 \%$ did not complete any sessions. Adherence analyses found that people with dementia who completed more sessions showed improved quality in the caregiving relationship and carers reported lower depressive symptoms at 26weeks. Qualitative data suggested that people with dementia and their carers experienced better communication as a result of iCST.

No studies have examined the impact of iCST in people with ID and dementia. Given the lack of previous data and potential issues with adherence rates to the iCST intervention, a feasibility study will help to address whether a full scale randomised controlled trial should be carried out in this population. 


\section{Adapting the intervention for use in people with ID}

We have modified and adapted the iCST manual in order to make it more suitable for use with people with ID and dementia. Where possible, we have retained the themes in the original manual but simplified the activities. However, some of the more complex activities were completely removed and substituted with alternative activities. An initial draft was developed with the input of a speech and language therapist. We then made further revisions to the manual following feedback from three group consultations with 12 health and social care professionals working with people with ID, 5 carers of people with ID and 5 individuals with ID. Selected activities from the manual were field-tested with five dyads (carer and individual with ID and dementia) who were asked to provide feedback on five activities each. Further changes to the manual were made based on the feedback.

\section{AIMS AND OBJECTIVES}

The aim of the study is to assess the feasibility of carrying out a future randomised controlled trial of iCST compared with TAU in people with ID and dementia. The primary objective of the study is to determine the feasibility of the intervention and study procedures, by assessing the recruitment rate and dropout rate of dyads (carer and individual with ID and dementia), the appropriateness of the outcome measures, adherence to the iCST intervention and the acceptability of the intervention.

The secondary objective of the study is to examine the effects of iCST on the outcome measures which include measures of cognitive and adaptive functioning and quality of life in individuals with dementia and measures of carer burden, competence and anxiety and depression in carers. In addition, we aim to estimate the sample size of a full scale randomised controlled trial.

\section{METHODS AND ANALYSIS \\ Design}

This will be a single blind, feasibility randomised controlled trial of iCST delivered by carers (formal or informal) versus TAU for people with ID and dementia. TAU has been selected as the comparator arm as it reflects current practice. Forty dyads (one carer and one individual with ID and dementia) will be randomised to either the intervention group or control group (TAU). Each arm will have 20 dyads. The primary and secondary outcomes will be measured at baseline prior to randomisation, at midpoint (11 weeks) and at the end of the intervention (21 weeks).

\section{Sample size}

A sample size of 40 has been selected for pragmatic reasons. Assuming a recruitment rate of $80 \%$ from participants who are eligible, a sample size of 40 provides a $95 \%$ CI for the recruitment rate of $67.6 \%-92.4 \%$. Assuming that $20 \%$ of participants drop out of the study, a sample size of 40 provides a $95 \%$ CI for the dropout rate of $7.60 \%-32.40 \%$.

\section{Participants}

\section{Inclusion criteria}

Participants will be over the age of 40 . This age has been selected as people with Down syndrome are likely to present with dementia from the age of 40 onwards (cases in younger people are less common). They will have premorbid mild or moderate ID and have a confirmed diagnosis of mild or moderate dementia. The participants will be screened for the presence of dementia using the International Classification of Diseases, 10th version (ICD-10) criteria (taken from the Cambridge Examination of Mental Disorders of Older People with Down's Syndrome and Others with Intellectual Disabilities (CAMDEX-DS). ${ }^{19}$ The CAMDEX-DS does not screen for the severity of dementia which will be assessed using ICD-10 criteria and clinical judgement. Mild dementia is defined as memory loss affecting the learning of new material, and the cognitive difficulties do not significantly impact on the person's ability to live independently. As most people with ID require some support with daily living, in someone with mild dementia and ID, the level of decline in functioning would not be expected to be severe enough to require a significant increase in the support they are receiving. In a person with ID and moderate dementia, the memory loss is more marked, and there is a significant decline in the ability of the individual to carry out activities of daily living that they were able to do previously, resulting in greater dependence on others.

The participants will need to be able to communicate verbally and in English, and be able to participate in simple games. Participants taking dementia medication can continue to take these during the study.

Each individual will also need to have a carer such as a member of staff, family member or friend who knows the individual well and is willing to take part in the study. Carers will need to be over the age of 18 , be able to speak English and provide consent to taking part.

\section{Exclusion criteria}

Participants will be excluded if they have severe dementia (indicated by a significant deterioration in cognitive functioning resulting in complete reliance on others and inability to recognise familiar people), significant physical illness or disability, visual or hearing impairment, or behavioural problems that could affect participation in the iCST sessions or during assessments.

\section{Recruitment}

Participants will be recruited from community learning (intellectual) disability teams based in England. Clinicians will be asked to screen their case load for potential participants (individuals with dementia and their carers) and will approach them to discuss the study. If they are interested in taking part, their details will be passed on to the trial research team who will contact the participant and their carer to arrange a face-to-face meeting in order 
to assess eligibility. If they are eligible, and the individual and their carer agree to take part, then informed consent will be obtained from both the carer and the participant with dementia.

Participants' capacity to consent to take part in the research will be assessed by the research assistant who will follow the guidelines stipulated in the Mental Capacity Act (2005). If the participant with dementia lacks the capacity to consent, a personal consultee (a relative or friend) will be consulted to consider the participant's beliefs and wishes about taking part in the study, and they will need to sign the declaration form before the participant is included in the study. If a personal consultee is not available, then we will consider approaching a nominated consultee (a member of the clinical team not directly involved in the research) who will need to sign a declaration form agreeing to the individual's participation in the study.

We estimate that we will need to recruit four eligible dyads each month, over 10 months. If recruitment is anticipated to be slow, we will recruit from other centres if necessary.

\section{Randomisation}

Randomisation will occur after eligibility, consent and baseline assessments have been carried out. Randomisation will be undertaken centrally by the coordinating trial team using a web-based system called Sealed Envelope. An administrator, who is not involved in the study, will enter the patient's trial ID into the web-based randomisation system (Sealed Envelope). This system will randomly allocate the participant to either the intervention or control arm, and he/she will inform the participants of their allocation. Randomisation will be based on varying block sizes. Although participants cannot be blinded to their allocated group, the research assistant administering the questionnaires will be blind to the allocation group. Due to the risk of carers revealing the allocation group, carers will be reminded before the follow-up assessments not to divulge this information. At the end of the study, we will assess researcher blindness by asking them to guess the allocated group.

\section{Intervention group}

The intervention will comprise 40 sessions of iCST which will be delivered by carers using the modified manual. The number of sessions has been reduced to 40 from the original 75 sessions in an attempt to improve adherence and acceptability of the intervention. Each carer will administer the activities within the manual two times a week for $30 \mathrm{~min}$, over a period of 20 weeks. Each session will begin with discussion of the day, date, weather and location ( $5 \mathrm{~min}$ ) followed by discussion of events in the news or current issues ( $5 \mathrm{~min}$ ) and then the main activity (20 min). The activities are based around a different theme for each session and have been designed to be fun and engaging for the individual, as well as mentally stimulating. Activities include word and number games, discussion of current affairs and famous people, creative and physical activities and quizzes. Carers will be encouraged to make the activities person-centred and multisensory and to tailor the activities to the ability and interests of the individual with ID. For example, pictures in the manual that the individual with ID is not familiar with could be replaced by pictures that are of interest or of relevance to them. Examples of how activities could be made easier or more challenging will be discussed.

The themes and activities within the manual are summarised in table 1.

\section{Carer training and support}

Carers will attend a half day training session on how to use the manual in either a group setting or will receive individual training at home, depending on their preference, and this will be provided by the research team. They will receive a copy of the adapted manual which includes paper-based activities and additional materials for specific activities (eg, dominoes, activity Compact Disc, dice).

Carers will be asked to keep a record of their sessions (eg, the duration, activities completed, level of engagement and enjoyment of activities by the individual and reasons for not competing the session) in a diary. In order to assess adherence to the manual, for each individual dyad, two sessions will be audio-taped (40 in total). A brief adherence measure will be developed for the study. If a carer is unable to continue the intervention (eg, due to poor health) then another carer can be substituted. In order to support the carers and to ensure continued momentum, the research team will contact carers at least once a month by phone and there will be regular contact by email. Home visits can also be carried out if needed. The intervention group will also have access to "usual care' and therefore the intervention arm will be examining the additional effects of iCST.

\section{Control group}

The control group will continue to have access to their usual care which will include anticholinesterase inhibitors, input from health professionals and any day activities. If they are interested, participants and carers in the control group will also be offered a copy of the manual and training in how to use it after the 20-week study period.

\section{Outcome measures}

The primary outcome: feasibility measures

Recruitment rate

We will assess the proportion of people who are referred to the study and are eligible to take part, and the proportion of people who are eligible and are willing to take part in the study. Reasons for refusing to take part in the study will be noted.

\section{Retention and dropout rate}

We will record the number of participants who completed assessments at each of the follow-up points and reasons for withdrawal/non-completion. 


\begin{tabular}{|c|c|c|c|}
\hline Session number & Themes/activities & Session number & Themes/activities \\
\hline 1 & My life & 21 & Associated words \\
\hline 2 & Food 1 & 22 & Orientation 1 \\
\hline 3 & Current affairs & 23 & Thinking cards \\
\hline 4 & Number games 1 & 24 & Household objects 1 \\
\hline 5 & Art discussion 1 & 25 & Categorising objects 1 \\
\hline 6 & Cross words & 26 & Number games 3 \\
\hline 7 & Physical games & 27 & Sounds 2 \\
\hline 8 & Childhood toys & 28 & Jobs \\
\hline 9 & Word games 1 & 29 & Scenes 2 \\
\hline 10 & Sound games 1 & 30 & Food 2 \\
\hline 11 & Using money & 31 & Art discussion 3 \\
\hline 12 & Travel & 32 & Household objects 2 \\
\hline 13 & Being creative & 33 & Physical games 2 \\
\hline 14 & Quiz games 1 & 34 & Orientation 2 \\
\hline 15 & Clothes & 35 & Signs and symbols \\
\hline 16 & Word games 2 & 36 & Word games 3 \\
\hline 17 & Scenes 1 & 37 & Memories of the past \\
\hline 18 & Number games 2 & 38 & Animals \\
\hline 19 & Brands and products & 39 & Categorising objects 2 \\
\hline 20 & Art discussion 2 & 40 & Quiz games 2 \\
\hline
\end{tabular}

\section{Appropriateness of outcome measures}

Missing data for each outcome measure will be analysed, as well as sensitivity of the outcome measure to change as a result of the intervention.

\section{Adherence to the intervention and acceptability of the intervention}

This will be assessed in a process evaluation using a mixed methods approach.

\section{Secondary outcome measures}

Outcome measures will be recorded in both individuals with dementia and their carer. at baseline, midway (11 weeks) and postintervention (21 weeks). See table 2 for the schedule of assessments.

\section{Outcomes in individuals with dementia}

Measures of cognition

Change in cognitive functioning will be measured by the Cambridge Cognitive Examination for older Adults with Down Syndrome (CAMCOG-DS) which will be administered with the individual with dementia. ${ }^{19}$ It includes an assessment of orientation, language, attention, praxis and abstract thinking. It provides individual subscale scores and total scores. Higher scores indicate better ability.

The Modified Memory for Objects test from the Neuropsychological Assessment of Dementia in Intellectual Disabilities Battery ${ }^{20}$ will also be administered with individuals with dementia. This assessment involves presenting seven every-day items to the individual and testing his/ her ability to recall an item that has been covered up. The maximum score is 7 . Higher scores indicate better ability.

The Cognitive Scale for Down Syndrome (CSDS) ${ }^{21}$ will be administered with carers. This measure has 61 items that have been validated in adults with Down syndrome but the items are relevant to people with ID in general. The scale includes items testing executive functioning, memory and language. Higher scores indicate better cognitive functioning.

Other outcome measures

Functional ability will be measured using the Alzheimer's Dementia Cooperative Study-Activities of Daily Living Inventory (ADCS-ADL). ${ }^{22}$ This will be administered with the carer. This is a measure of the ability of the individual with dementia to carry out a range of daily activities. There are 23 items covering a range of areas such as feeding, bathing, grooming, preparing meals, use of household appliances and hobbies. The maximum score is 78. Higher scores indicate better ability. This measure has not been validated in people with ID but has been found to be sensitive to change after CST. ${ }^{23}$ Most of the items appear to be relevant to the ID population.

Quality of life will be assessed using the Quality of Life-Alzheimer's Disease Scale (QOL-AD) ${ }^{24}$ This will be administered with the carer. This is a 13-item scale with items covering physical health, mood, family life and functioning. The maximum score is 52, with 
Table 2 Schedule of assessments

\begin{tabular}{|c|c|c|c|c|c|c|c|}
\hline \multirow[b]{2}{*}{ Visit no } & \multirow{2}{*}{$\begin{array}{l}\begin{array}{l}\text { Screening } \\
\text { (pretreatment } \\
\text { assessment) }\end{array} \\
1\end{array}$} & \multicolumn{4}{|c|}{ Intervention phase } & \multirow{2}{*}{$\begin{array}{l}\text { Final visit } \\
6\end{array}$} & \multirow{2}{*}{$\begin{array}{l}\text { Optional } \\
7\end{array}$} \\
\hline & & 2 & 3 & 4 & 5 & & \\
\hline Week number: & & 1 & 5 & 11 & 15 & 20 & \\
\hline Window of flexibility for timing of visits: & & & $\begin{array}{l}\text { Eg, } \\
\pm 7 \text { days }\end{array}$ & $\begin{array}{l}\text { Eg, } \\
\pm 7 \text { days }\end{array}$ & & $\begin{array}{l}\text { Eg, } \\
\pm 7 \text { days }\end{array}$ & \\
\hline Informed consent & $x$ & & & & & & \\
\hline Medical history & $x$ & & & & & & \\
\hline Eligibility confirmation (ICD-10 criteria) & $x$ & & & & & & \\
\hline CAMCOG-DS & $x$ & & & $x$ & & $x$ & \\
\hline Memory for objects test & $x$ & & & $x$ & & $x$ & \\
\hline CSDS & $x$ & & & $x$ & & $\mathrm{x}$ & \\
\hline ADCS-ADL & $x$ & & & $x$ & & $x$ & \\
\hline QOL-AD & $\mathrm{x}$ & & & $\mathrm{x}$ & & $\mathrm{x}$ & \\
\hline Caregiver Burden Scale & $x$ & & & $x$ & & $x$ & \\
\hline HADS & $x$ & & & $\mathrm{x}$ & & $\mathrm{x}$ & \\
\hline SCIDS Scale & $x$ & & & $x$ & & $x$ & \\
\hline Training for carers & & $\mathrm{x}$ & & & & & \\
\hline Treatment adherence/monitoring visit & & & $x$ & & $x$ & & \\
\hline Trial evaluation questionnaire & & & & & & $x$ & \\
\hline Semistructured interview & & & & & & & $x$ \\
\hline Randomisation & $\mathrm{x}$ & & & & & & \\
\hline Adverse events review & $x$ & $x$ & & $x$ & & $x$ & \\
\hline Concomitant medication review & $\mathrm{x}$ & $x$ & & $\mathrm{x}$ & & $x$ & \\
\hline
\end{tabular}

ADCS-ADL, Alzheimer's Dementia Cooperative study-Activities of Daily Living Inventory; CAMCOG-DS, The Cambridge Cognitive Examination for older Adults with Down Syndrome; CSDS, Cognitive Scale for Down Syndrome; HADS, Hospital Anxiety and Depression Scale; QOL-AD, Quality of life-Alzheimer's Disease Scale; SCIDS, Sense of Competence in Dementia Care Staff.

higher scores indicating a better quality of life. There are currently no suitable measures for quality of life in people with ID and dementia. This measure has been used in studies of CST as a primary outcome measure and has been found to detect an improvement in quality of life. $^{12} 23$

\section{Carer outcomes}

Care giving burden in both paid and informal carers will be assessed using the Care Giving Burden Scale (SCIDS) ${ }^{25}$ Carers will be asked if the individual requires assistance in a range of areas, whether they have provided assistance in the last month and whether providing assistance has been stressful. There are three domains and each has a maximum score of 15 .

The competence to look after someone with dementia will be assessed using the Sense of Competence in Dementia Care Staff Scale. ${ }^{26}$ This is a 17 -item scale with four subscales (professionalism, building relationships, care challenges and sustaining personhood). The maximum score is 68 , with higher scores indicating more competence. Although this questionnaire was developed for care staff, the questions may also be relevant for family members. Minor modifications to the questions will need to be made to ensure that it is appropriate for use in both groups. This measure has been found to be sensitive to change following $\mathrm{CST}^{27}$

The presence of an anxiety or depressive disorder in the carer will be assessed using the Hospital Anxiety and Depression Scale (HADS) ${ }^{28}$

\section{Process evaluation}

A process evaluation will be carried out, based on Medical Research Council guidance. ${ }^{29}$ The aim of the process evaluation will be to examine whether the different components of the intervention (eg, training of carers, monitoring visits) were consistently followed; the extent to which iCST is delivered as intended; the extent to which the intervention would need to be modified prior to a full trial in order to make it more acceptable to participants and understanding the perceived value, benefits and harm or unintended consequences of the intervention so that these are fully measured in the full trial. In order to carry out the process evaluation, a mixed methods approach employing qualitative and quantitative approaches will be used. 


\section{Qualitative approach}

At the end of the intervention, all the participants will be given a short questionnaire to complete about their experience and satisfaction in participating in the study and to provide feedback. Participants will be asked whether they thought the intervention was practical and acceptable, such as whether the length and number of sessions were appropriate. In addition, five dyads from the intervention group and five dyads from the control group will also be invited to participate in a semistructured interview where more detailed feedback will be obtained in relation to the acceptability of the intervention. We will enquire about what aspects of the intervention worked well or could be further improved and whether there were any positive or negative effects of the intervention on the individual with dementia. Participants will also be asked about their experience of the study processes (eg, randomisation) and assessments. The interviews will be audio-taped and transcribed verbatim. Transcripts will be analysed using thematic analysis supported by computer software (NVivo V.9). The analytic strategy will identify themes relating to the barriers and facilitators that will enable the successful implementation of the intervention.

\section{Quantitative approach}

Carer diaries will be examined to identify the number of sessions that were completed by each dyad and reasons for non-completion. We will calculate the proportion of dyads who were able to complete all the sessions, those who were able to complete $50 \%$ and those who did not complete any. We will examine the feedback for each activity to identify which themes and activities were likely to be completed or missed. The extent of participation of the individuals in the sessions will also be examined (eg, full or partial participation). The quality of the delivery of the iCST intervention will also be assessed by audiotaping two sessions carried out by each dyad (40 in total) and these will be rated on the extent to which they follow the manual.

One of the major challenges will be changes in paid carers or periods where no carer may be available due to illness or annual leave during the course of the intervention. We will examine the frequency of changes in carers and the number of sessions missed due to unavailability of carers. We will examine whether there are differences in adherence rates between paid and family carers.

\section{Incentives}

In both arms of the study, the carers and participants with dementia will receive a $£ 10$ gift voucher for completing each follow-up assessment (at 11 weeks and 21 weeks; £20 in total). Carers in the intervention arm will receive an additional $£ 10$ gift voucher for competing each audiotaped session (£20 total). The participants who take part in the postintervention interviews will also receive a $£ 10$ gift voucher each.

\section{Patient and public involvement}

Patients and the public were not involved in the design of the study and will not be involved in the conduct of the study. The findings of the study will be disseminated to the study participants in the form or an accessible newsletter.

\section{Statistical analysis}

The study sample will be analysed using descriptive statistics. Data on recruitment will be recorded and examined. This will include information on how many participants were approached and agreed to be screened; how many met the eligibility criteria and agreed to take part and how many completed the study, or dropped out. This information will be presented in a Consolidated Standards of Reporting Trials (CONSORT) diagram describing the flow of participants through the study (see figure 1).

The baseline clinical and demographic characteristics of participants in both arms of the study will be compared descriptively. Descriptive statistics (mean and SD), and the proportion of participants who competed the measures, will be reported for each outcome at baseline, 11 weeks and 21 weeks which will provide data on determining the most appropriate outcome for a future trial.

A linear regression model adjusting for baseline scores will be used to estimate the effect of iCST on each of the outcome variables. The results will be presented as estimates with $95 \%$ CIs. The analysis will be based on intention to treat and will be exploratory due to the small sample size. Based on this analysis, an appropriate primary outcome will be identified and will be used to estimate the sample size for a future RCT.

\section{Criteria for progression to a full trial}

We will consider a full trial if the following criteria are met:

1. If we are able to achieve at least $70 \%$ of our recruitment target of 40 (28 dyads or more).

2. If $75 \%$ of the dyads in the intervention arm complete at least half the number of sessions (20 sessions out of $40)$. If less than $50 \%$ of the dyads complete half the sessions, the intervention is likely to be ineffective ${ }^{16}$

3. The dropout rate of dyads in the entire study is less than $30 \%$.

4. The intervention and trial procedures are considered to be acceptable by study participants.

If the study recruits 50-69\% of the target, 50-74\% complete at least half the number of sessions and the drop out rate is $21-30 \%$ we will consider whether measures can be implemented in order to improve these outcomes and this will inform our decision to progress to a full trial. If the recruitment rate is below $50 \%$ and less than $50 \%$ complete half the sessions and the drop out rate is more than $30 \%$, we will not consider running a full trial.

\section{ETHICS AND DISSEMINATION}

\section{Research governance and trial sponsorship}

Any amendments to the trial protocol, participant information sheets, consent forms, General Practitioner letters 


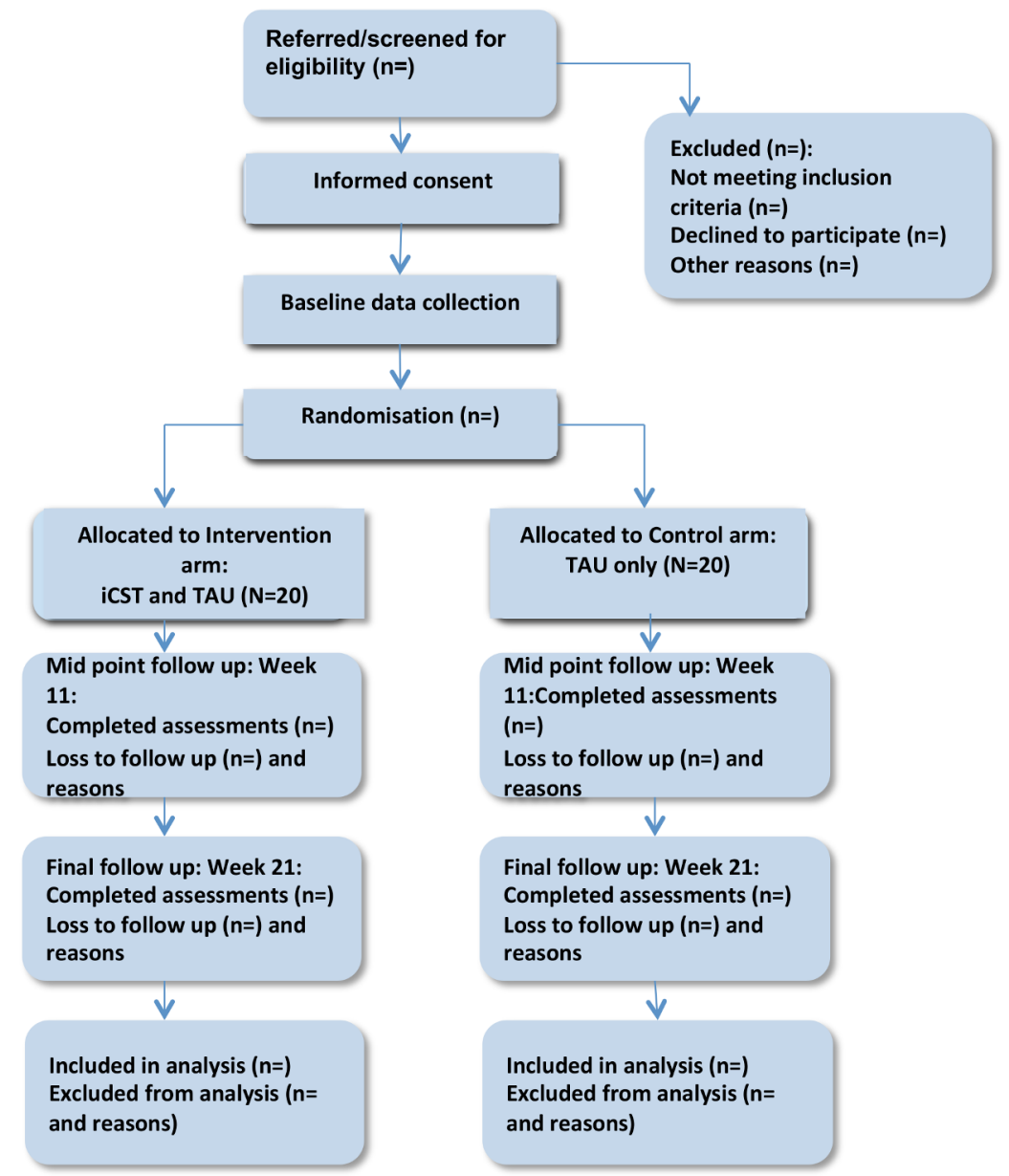

Figure 1 Trial flow chart illustrating the flow of participants through the study from referral through to analysis of data. The number of participants who are referred and are eligible will be recorded as will the number of people who are eligible and agree to be randomised. Reasons for participant withdrawal/dropout will be recorded. iCST, individual cognitive stimulation therapy; TAU, treatment as usual.

and any submitted supporting documents will not be implemented prior to receipt of the required approvals. Paper-based data that are collected will be stored securely in locked filing cabinets in locked offices at University College London. Participants will be given participant identification numbers, and participant identifiable data will be anonymised and password protected. The results of the study will be published in peer-reviewed journals and presented at conferences.

\section{Study time line}

The trial is anticipated to last a duration of 18 months. There will be 10 months of recruitment and a further five months for follow up assessments. There will be three months to complete the process evaluation, to analyse the results and write up the study findings.

\section{DISCUSSION}

Dementia is more common in people with ID but the evidence base for interventions in dementia in people with ID is very limited. iCST may be particularly useful in people with ID due to the person-centred nature of the intervention, making it easier to tailor activities based on the individual's preference and the availability of paid carers who may be able to deliver the intervention as part of their caring role. To our knowledge, this is the first feasibility study of iCST in people with ID and dementia. The study will provide information about whether this treatment is feasible and acceptable for people with ID.

A key challenge that may arise from the study is adherence to the intervention. ${ }^{18}$ By reducing the number of sessions to be delivered by the carer from 70 to 40 , we hope to minimise the burden on carers delivering the intervention. Previous studies on iCST have been carried out using only family carers, whereas we intend to include paid carers. This may have an advantage in that paid carers are more likely to have dedicated time that they can use to carry out the iCST sessions (eg, as part of a key work session), and they may value the structured nature of the intervention and appreciate the therapeutic value of the intervention in enhancing communication and interaction with the individual. However, not all individuals will have access to one-to-one support from a paid carer, even if they live in a care home, and paid carers may also experience stress as a consequence of caring for 
someone with dementia. ${ }^{30}$ Other challenges include the frequent changes and turnover of staff which could affect the delivery of the intervention and the value of having carer outcome measures.

If the study meets the prespecified progression criteria, we will apply for funding to conduct a full-scale RCT in order to examine the effectiveness of iCST compared with TAU. This study could lead to changes in health policy, including improved access to CST for people with ID and dementia.

Acknowledgements The authors would like to thanks Richard Lohan for assisting with the preparation of the study documentation and Jack Gaughan and Ambrose Viall for their input into the development of the manual.

Contributors AA is the chief Investigator of the study and is responsible for ensuring that the study follows the agreed protocol. AA, AH, EA and AS were involved in the conceptualisation of the study and are part of the trial management group. EB is a research assistant who has been involved in the development of the manual, and will be carrying out the baseline and outcome assessments. All the authors were involved in writing and reviewing the contents of the paper. AA will have access to the final data set.

Funding This research is being supported by the Baily Thomas Charitable Fund (reference number TRUST/NC/AC/SG/3755-6846). The study is being sponsored by University College London.

Competing interests None declared.

Patient consent Will be carried out according to the Mental Capacity Act (2005).

Ethics approval Ethics approval was obtained from the Harrow Research Ethics Committee on 20/03/2017 (reference: 17/L0/0030), for the modification phase of the intervention, as well as for the trial. Health Research Authority approval was also obtained on 11/05/2017.

Provenance and peer review Not commissioned; externally peer reviewed.

Open access This is an open access article distributed in accordance with the Creative Commons Attribution Non Commercial (CC BY-NC 4.0) license, which permits others to distribute, remix, adapt, build upon this work non-commercially, and license their derivative works on different terms, provided the original work is properly cited, appropriate credit is given, any changes made indicated, and the use is non-commercial. See: http://creativecommons.org/licenses/by-nc/4.0/.

\section{REFERENCES}

1. Strydom A, Chan T, King M, et al. Incidence of dementia in older adults with intellectual disabilities. Res Dev Disabil 2013;34:1881-5.

2. McCarron M, McCallion P, Reilly E, et al. A prospective 20-year longitudinal follow-up of dementia in persons with Down syndrome. $J$ Intellect Disabil Res 2017;61:843-52.

3. Englund A, Jonsson B, Zander CS, et al. Changes in mortality and causes of death in the Swedish Down syndrome population. Am J Med Genet A 2013;161A:642-9.

4. Bahar-Fuchs A, Clare L, Woods B. Cognitive training and cognitive rehabilitation for mild to moderate Alzheimer's disease and vascular dementia. Cochrane Database Syst Rev 2013;5:CD003260.

5. Clare L, Linden DE, Woods RT, et al. Goal-oriented cognitive rehabilitation for people with early-stage Alzheimer disease: a singleblind randomized controlled trial of clinical efficacy. Am J Geriatr Psychiatry 2010;18:928-39.

6. Woods B, O'Philbin L, Farrell EM, et al. Reminiscence therapy for dementia. Cochrane Database Syst Rev 2018;3:CD001120.

7. Spector A, Orrell M, Davies S, et al. Can reality orientation be rehabilitated? Development and piloting of an evidence-based programme of cognition-based therapies for people with dementia. Neuropsychol Rehabil 2001;11:377-97.
8. Spector A, Thorgrimsen L, Woods B, et al. Making a difference: an evidence-based group programme to offer cognitive stimulation therapy (CST) to people with dementia (manual for group leaders). London: Hawker, 2006.

9. Woods B, Aguirre E, Spector AE, et al. Cognitive stimulation to improve cognitive functioning in people with dementia. Cochrane Database Syst Rev 2012;2:CD005562.

10. Spector A, Orrell M, Woods B. Cognitive Stimulation Therapy (CST): effects on different areas of cognitive function for people with dementia. Int J Geriatr Psychiatry 2010;25:1253-8.

11. Hall L, Orrell M, Stott J, et al. Cognitive stimulation therapy (CST): neuropsychological mechanisms of change. Int Psychogeriatr 2013;25:479-89.

12. Spector A, Thorgrimsen L, Woods B, et al. A randomised controlled trial investigating the effectiveness of an evidence-based cognitive stimulation therapy programme for people with dementia. $B J$ Psychiatry 2003;183:248-54.

13. National Institute for Health and Clinical Excellence and the Social Care Institute for Excellence (NICE-SCIE). Dementia: supporting people with dementia and their carers in health and social care Clinical Guideline 42. London: NICE-SCIE, 2006.

14. Knapp M, Thorgrimsen L, Patel A, et al. Cognitive stimulation therapy for people with dementia: cost-effectiveness analysis. Br J Psychiatry 2006;188:574-80.

15. Shanahan SF. Efficacy of a cognitive stimulation therapy programme with adults with Down syndrome: a randomised study Thesis (D.Clin. Psych.), University of Essex. 2014 http://ethos.bl.uk/OrderDetails. do?uin=uk.bl.ethos.617081

16. Yates L, Orrell M, Leung P, et al. Making a difference 3: individual cognitive stimulation therapy: a manual for carers: Hawker publications, 2014

17. Onder G, Zanetti O, Giacobini E, et al. Reality orientation therapy combined with cholinesterase inhibitors in alzheimer's disease: randomised controlled trial. Br J Psychiatry 2005;187:450-5.

18. Orrell $M$, Yates $L$, Leung $P$, et al. The impact of individual Cognitive Stimulation Therapy (iCST) on cognition, quality of life, caregiver health, and family relationships in dementia: a randomised controlled trial. PLoS Med 2017;14:e1002269.

19. Ball SL, Holland AJ, Huppert FA, et al. CAMDEX-DS: The Cambridge examination for mental disorders of older people with down's syndrome and others with intellectual disabilities. Cambridge: Cambridge University Press, 2006.

20. Oliver $C$, Crayton L, Holland A, et al. A four year prospective study of age-related cognitive change in adults with Down's syndrome. Psychol Med 1998;28:1365-77.

21. Startin CM, Rodger E, Fodor-Wynne L, et al. Developing an informant questionnaire for cognitive abilities in down syndrome: the Cognitive Scale for Down Syndrome (CS-DS). PLoS One 2016;11:e0154596.

22. Galasko D, Bennett $D$, Sano M, et al. An inventory to assess activities of daily living for clinical trials in alzheimer's disease. Alzheimer Disease \& Associated Disorders 1997;11:33-9.

23. Thorgrimsen L, Selwood A, Spector A, et al. Whose quality of life is it anyway? The validity and reliability of the Quality of Life-Alzheimer's Disease (QoL-AD) scale. Alzheimer Dis Assoc Disord 2003;17:201-3.

24. Orrell M, Aguirre E, Spector A, et al. Maintenance cognitive stimulation therapy for dementia: single-blind, multicentre, pragmatic randomised controlled trial. Br J Psychiatry 2014;204:454-61.

25. Macera CA, Eaker ED, Jannarone RJ, et al. A measure of perceived burden among caregivers. Eval Health Prof 1993;16:204-11.

26. Schepers AK, Orrell M, Shanahan N, et al. Sense of competence in dementia care staff (SCIDS) scale: development, reliability, and validity. Int Psychogeriatr 2012;24:1153-62.

27. Streater A, Aguirre E, Spector A, et al. Cognitive stimulation therapy for people with dementia in practice: a service evaluation. British Journal of Occupational Therapy 2016;79:574-80.

28. Zigmond AS, Snaith RP. The hospital anxiety and depression scale. Acta Psychiatr Scand 1983;67:361-70.

29. Moore GF, Audrey S, Barker M, et al. Process evaluation of complex interventions: medical research council guidance. BMJ 2015;350:h1258.

30. Zimmerman S, Williams CS, Reed PS, et al. Attitudes, stress, and satisfaction of staff who care for residents with dementia. Gerontologist 2005;45 Spec No 1:96-105. 